\title{
Vinculación entre patrones de aprendizaje y patrones temporales en estudiantes universitarios argentinos
}

Linkage Between Learning Patterns and Temporary Patterns in Argentinean University Students

Ligação entre padrões de aprendizagem

e padrões temporais em estudantes

universitários argentinos

Mariela Lourdes González* iD orcid.org/0000-0002-2649-1579

Hilda Difabio de Anglat** (iD orcid.org/0000-0002-9679-1745

doi: 10.17227/rce.num77-9597

Para citar este artículo: Lourdes, M. y Difabio, H. (2019). Vinculación entre patrones de aprendizaje y patrones temporales en estudiantes universitarios argentinos. Revista Colombiana de Educación, 77, 277-298. doi: 10.17227/rce.num77-9597

\section{(c) $\underset{\mathrm{BY}}{(\text { ) }(9)}$}

* Universidad Nacional de Cuyo, Facultad de Filosofía y Letras, Mendoza, Argentina. Correo electrónico: marielalourdesgonzalez@yahoo.com.ar.

** Universidad Nacional de Cuyo-Consejo Nacional de Investigaciones Científicas y Técnicas (Conicet), Mendoza, Argentina. Correo electrónico:hdifabio@mendoza-conicet.gob.ar 


\section{Resumen}

El presente estudio tiene por objetivos: identificar los patrones de aprendizaje y patrones temporalesen una muestra de estudiantes universitarios argentinos, relacionar los patrones de aprendizaje con la perspectiva temporal en estudiantes universitarios, identificar diferencias según variables personales (género y edad) y contextuales (carrera y curso). En un primer momento, se presentan los fundamentos teóricos de los constructos mencionados. En cuanto a la metodología, en situación regular de clase se aplicó el Inventory Learning Styles (ıs) de Jan Vermunt en la versión española, traducida y validada por Martínez-Fernández et al. (2009), y luego se administró el Zimbardo Time Perspective Inventory (ZTPI) de Zimbardo y Boyd (1999), en la adaptación al español de Díaz-Morales (2006a y 2006b). Se presentan los resultados obtenidos en una muestra $(N=616)$ de alumnos de la Universidad Nacional de Cuyo. Estos arrojan diferencias significativas por curso y edad, así como en la conformación de tres patrones temporales que se combinan con los cuatro patrones de aprendizaje postulados por Vermunt (1998).

\section{Palabras clave}

Aprendizaje; factor tiempo; estudiantes universitarios

\section{Keywords}

Learning; time factor; university students

\begin{abstract}
The present study aims at: identifying learning patterns and temporal patterns in a sample of Argentine university students, relating learning patterns to the tempora perspective in university students, and identifying differences according to personal variables (gender and age) and contextual variables (career and course). At first, the authors present the theoretical foundations of the above mentioned constructs. Then, they show the results obtained in a sample $(N=616)$ of students of the National University of Cuyo. Regarding the methodology, in a regular class situation, the authors applied the Spanish version of Jan Vermunt's Inventory of Learning Styles (ILS), translated and validated by Martínez-Fernández et al. (2009), and administered Díaz-Morales' (2006a and 2006b) Spanish adaptation of the Zimbardo Time Perspective Inventory (ZTPI), developed by Zimbardo and Boyd (1999). The results show significant differences by course and age, as well as in the conformation of three temporal patterns that are combined with the four learning patterns postulated by

Vermunt (1998)
\end{abstract}

\section{Resumo}

Este estudo tem como objetivos: 1) identificar padrões de aprendizagem e padrões temporais em uma amostra de estudantes universitários argentinos, 2) relacionar os padrões de aprendizagem com a perspectiva temporal em estudantes universitários, 3) identificar as diferenças de acordo com variáveis pessoais (sexo e idade) e contextuais (carreira e curso). Inicialmente, são apresentados os fundamentos teóricos dos construtos supracitados; em seguida, os dados obtidos numa amostra $(\mathrm{N}=616)$ dos estudantes da Universidade Nacional de Cujo. Em relação à metodologia, o Inventory Learning Styles (ıs) de Jan Vermunt foi aplicado em uma situação de classe regular na versão em espanhol, traduzida e validada por Martínez-Fernández et al. (2009), e depois o Zimbardo Time Perspective Inventory (ZTPI) de Zimbardo e Boyd (1999) foi administrado na adaptação espanhola de Díaz-Morales (2006a e 2006b). Os resultados mostram diferenças significativas por curso e idade, bem como na conformação de três padrões temporais que são combinados com os quatro padrões de aprendizagem postulados por Vermunt (1998).

\section{Palavras-chave}

Aprendizagem; fator tempo; estudantes universitários 


\section{Introducción}

La investigación acerca de los estilos, enfoques o patrones de aprendizaje — denominaciones en uso que responden a distintos encuadres teóricosconstituye hoy un corpus extensamente desarrollado con varios modelos e instrumentos (cfr., entre muchos otros, Aguilar-Rivera, 2010; Entwistle, McCune y Walker, 2001; García-Fuentes, Muñoz-Cantero y Abalde-Paz, 2002; Marín Gracia, 2002). El término estilo, empleado en un principio por el autor del modelo que se sigue en este trabajo, Jan Vermunt, podía dar lugar a que se lo entendiera como un atributo invariante, un rasgo de personalidad; por esa razón, lo reemplazó por el de pattern, esto es, 'patrón' (Vermunt, 1996, 1998, 2005), que alude a una forma de estudiar y de considerar la actividad de aprendizaje relativamente estable pero no inmutable.

Por otra parte, en los últimos años, un número considerable de estudios destaca la incidencia de la perspectiva temporal (PT) en el aprendizaje y, específicamente, en su autorregulación (De Bilde, Vansteenkiste y Lens, 2011; Mclnerney, 2004; Suddendorf y Busby, 2005; Zebardast et al., 2011) porque la previsión de metas a largo plazo y el aumento de la instrumentalidad de la conducta presente se asocian con motivación enérgica, perseverancia intensiva durante el proceso intelectual, aplicación de estrategias apropiadas y aprendizaje profundo (Simons, Vansteenkiste, Lens y Lacante, 2004). En este sentido, se ha probado que la perspectiva temporal futura (PTF) conduce a un mayor compromiso académico (Horstmanshof y Zimitat, 2007; Peetsma, 2000) y a un mejor rendimiento escolar y profesional (Adelabu, 2007; De Bilde et al., 2011; Gutiérrez-Braojos, Salmerón-Pérez y Muñoz-Cantero, 2014; Peetsma, Hascher, Van der Veen y Roede, 2005; Shell y Husman, 2001). Asimismo, se la ha relacionado con variables personales —por ejemplo, género (Brenlla, Willis y Germano, 2016; Greene y De Backer, 2004)—, contextuales y con la edad (Laureiro-Martinez, Trujillo y Unda, 2017).

No obstante, algunos autores (Worrell y Mello, 2007; Zimbardo y Boyd, 1999) señalan la conveniencia de analizar todas las perspectivas temporales, ya que "el énfasis en el futuro ha resultado en un descuido relativo de la importancia potencial de las perspectivas temporales pasada y presente" (Worrell y Mello, 2007, p. 488).

Entre los estudios que buscan establecer explícitamente la vinculación de los patrones de aprendizaje con la perspectiva temporal, aunque constreñida al aprendizaje autorregulado, se destacan el modelo teórico que elaboran Miller y Brickman (2004) y la investigación empírica de Gutiérrez-Braojos, Salmerón-Pérez y Muñoz-Cantero (2014). 
Los primeros, en la explicación de su modelo, parten de ponderar el papel de la meta - "una representación del futuro" de extensión variable (Miller y Brickman, 2004, p. 14) — en el engranaje autorregulatorio: en primer lugar, define el resultado que esperamos lograr; en segundo lugar, pone en foco aspectos del desempeño que debemos observar y controlar durante el proceso de aprendizaje; por último, se constituye en un criterio para la autoevaluación del resultado. Señalan que la persona, antes de comprometerse con una meta, sopesa la factibilidad de su logro, aunque ello no implica un juicio cuidadosamente deliberado (dado que es improbable que un análisis detallado de pasos futuros se produzca antes de algún grado de compromiso inicial con la meta), sino una ponderación global que se basa, por un lado, en las creencias generales de autoeficacia y en el autoconcepto sobre la habilidad en un determinado dominio, y, por otro, en la instrumentalidad observada en las tareas disponibles en cuanto dichas tareas se ponderan como oportunidades valiosas para lograr fines personalmente valorados (por ejemplo, carrera o trabajo, desarrollo de relaciones personales, contribución a la sociedad, etc.). En este sistema, solo el establecimiento de metas próximas para fines futuros promueve el compromiso con la tarea y la puesta en obra de estrategias específicas de resolución de problemas, ambos componentes necesarios para poner en marcha el proceso autorregulatorio (autobservación, autoevaluación, autorreacción, entre otros).

La investigación de Gutiérrez-Braojos et al. (2014), por su parte, halla tres patrones temporales (PT): proactivo (caracterizado por pasado positivo y perspectiva orientada al futuro), balanceado (equilibrio entre las tres orientaciones temporales), e interrumpido, "una orientación anclada en el presente con una tendencia hedonista y una interrupción en la construcción de representaciones temporales futuras" (pp. 282-283). Estos patrones tienen un efecto modulador sobre el logro académico a través de la autorregulación del aprendizaje, siendo el primero el que resulta más propio del aprendiz autorregulado y el último (el interrumpido), de aquel que requiere de una regulación externa. El aprendiz con PT balanceada, por su parte, muestra una combinación de estrategias reguladoras externas e internas.

En consecuencia, el presente estudio busca extender la investigación previa, ya que se propone analizar la vinculación existente entre patrones de aprendizaje y perspectivas temporales; esto es, relacionar el modelo de componentes del aprendizaje propuesto por Vermunt con el modelo de Zimbardo y Boyd, que pone especial énfasis en la dimensión temporal: pasado, presente y futuro. Por otra parte, se propone analizar la asociación entre ambos constructos $y$ factores personales (género y edad) y contextuales (carrera universitaria y año que se cursa). 


\section{Patrones de aprendizaje en el modelo de Vermunt}

Según Vermunt (2005), un patrón de aprendizaje se caracteriza por una combinación interrelacionada de componentes del aprendizaje, tales como sus concepciones y motivos para estudiar, las actividades que habitualmente desarrolla el estudiante y cómo regula los procesos.

En la clásica definición de Keefe, el estilo de aprendizaje está conformado por "factores cognitivos, afectivos y fisiológicos, que sirven como indicadores relativamente estables, de cómo los aprendices perciben, interaccionan y responden a sus ambientes educativos" (1985, p. 140). En cambio, Vermunt $(1996,1998,2005)$ conceptualiza el patrón de aprendizaje como un constructo que alude a una forma de aprender también relativamente estable pero no inmutable, a un interjuego de factores, variables personales y contextuales, que lleva implícita la posibilidad de desarrollo a través del tiempo.

El modelo incluye cuatro componentes: concepciones de aprendizaje (modelos mentales), orientaciones motivacionales, estrategias de procesamiento y de regulación. Las primeras se refieren a las redes de creencias, ideas, supuestos, acerca del conocimiento y el aprendizaje. Las orientaciones motivacionales son las metas personales, intenciones, expectativas, dudas, que un estudiante puede experimentar durante su formación. Las estrategias de procesamiento remiten a las actividades intelectuales que el alumno aplica para procesar el contenido del aprendizaje (por ejemplo, distinguir ideas principales y secundarias, relacionar aspectos de un área temática, elaborar ejemplos). Las estrategias de regulación son aquellas que el estudiante aplica para planificar la tarea, monitorear su desarrollo, diagnosticar las causas de los escollos y ajustar el proceso cuando sea necesario. Vermunt propone tres vías de regulación: 1) la autorregulación, 2) la regulación externa y 3) la ausencia de regulación. La primera hace referencia a la planificación, la supervisión y el control por parte del estudiante de sus procesos y resultados del aprendizaje, y a la regulación de contenidos. La segunda, alude a que el estudiante espera que la activación de estrategias devenga de otros (profesores, compañeros, tutores, familia). Y la tercera, tiene que ver con la falta de control, estrategias o acciones por parte del estudiante para hacer frente a las demandas o exigencias educativas.

Sobre la base de una extensa investigación empírica, Vermunt (1998) identificó cuatro patrones: dirigido al significado (meaning directed), dirigido a la aplicación, (application directed), dirigido a la reproducción (reproduction directed) y no dirigido (undirected). Para su caracterización, se los conceptualiza brevemente.

En el patrón dirigido al significado (MD), el estudiante emplea estrategias de procesamiento profundo (relación-estructuración, por ejemplo entre el nuevo contenido y el conocimiento previo, y pensamiento crítico para 
elaborar interpretaciones, opiniones y conclusiones propias), estrategias de regulación de los contenidos, procesos y resultados del aprendizaje (esto es, evalúa su progreso en el aprendizaje formulándose preguntas), su orientación es un interés personal en el tema que se aborda y su modelo mental implica la concepción del conocimiento como construcción del significado.

En el dirigido a la aplicación (AD), el estudiante encamina sus procesos intelectuales a aplicar lo aprendido en situaciones concretas, del mundo real — por ello, presta especial atención a los contenidos que evidencian relevancia práctica-, regula su aprendizaje tanto interna como externamente, tiene como meta principal aprender a utilizar el conocimiento que va incorporando a través del estudio en su futura actividad laboral.

En el patrón reproductivo (RD), se prioriza la memorización y el repaso a fin de aprobar las evaluaciones (la meta más importante del estudio). Se centra en las indicaciones del docente y del material de aprendizaje (regulación externa), su procesamiento es secuencial (parte por parte, cada tema por separado) y exhaustivo desde la concepción del aprendizaje como incorporación de conocimiento.

Finalmente, en el no dirigido (UD), el alumno experimenta dificultades para procesar el material de estudio - especialmente de la extensión y la complejidad frecuentes en la educación universitaria-, delega en el grupo o en el docente la función reguladora, suele manifestar baja autoeficacia y poca confianza en sus capacidades.

Para evaluarlos, Vermunt (1994) desarrolló el Inventory of Learning Styles (ILS), una escala conformada por 120 indicadores valorados en escalas Likert, del 1 al 5, que se construyó a partir del análisis fenomenográfico de entrevistas con estudiantes universitarios sobre sus modos de aprender y de regularse, sus creencias sobre el aprendizaje, el estudio y la enseñanza, y sus motivos, preocupaciones y metas personales en el estudio (Vermunt, 1996). Para los ítems sobre estrategias de procesamiento y de regulación, los estudiantes deben indicar en qué grado (desde "Lo hago rara vez o nunca" hasta "Lo hago siempre") prefieren utilizar, durante el estudio, las actividades referidas; para los indicadores relativos a las orientaciones y concepciones de aprendizaje, se les solicita que señalen el grado (desde "Totalmente en desacuerdo" hasta "Completamente de acuerdo") en que los puntos de vista y los motivos descritos se corresponden con sus propios puntos de vista y motivos.

\section{Perspectiva temporal}

Según Zimbardo y Boyd (1999), es Kurt Lewin quien revaloriza el papel de la PT en la conducta humana, definiéndola como "la totalidad de las visiones individuales sobre el propio futuro y pasado psicológicos en un tiempo dado" (Zimbardo y Boyd, 1999, p. 1271). Joseph Nuttin reafirmó el 
modelo lewiniano al señalar que "los eventos futuros y pasados impactan la conducta actual hasta el extremo de que están presentes en el nivel cognitivo del funcionamiento conductual" (1985, p. 54). También la teoría de la autoeficacia (Bandura, 1997) postula una influencia temporal tripartita en la autorregulación generada por el impacto de las creencias de eficacia, que se basan en las experiencias pasadas, las valoraciones actuales y la reflexión sobre las opciones futuras.

La PT es un fenómeno multidimensional (Nurmi, 1989), con frecuencia no consciente, ${ }^{1}$ aun cuando se adscriben las experiencias personales y sociales a categorías temporales, o marcos de tiempo, que contribuyen a proporcionar orden, coherencia y significado a dichos eventos; a zonas de tiempo por medio de las cuales los individuos fraccionan el fluir de sus experiencias temporales. Estos marcos cognitivos pueden reflejar patrones temporales cíclicos, recursivos, o eventos no recurrentes en la vida de una persona. Se emplean en la codificación, el almacenaje y el recuerdo de los eventos, así como en la formación de expectativas, metas y escenarios imaginativos. "Entre la construcción abstracta del pasado y la anticipación del futuro, descansa la representación concreta, empíricamente centrada, del presente" (Zimbardo y Boyd, 1999, p. 1271).

Zimbardo y Boyd (2008) postulan que estas perspectivas temporales aprendidas ejercen una influencia dinámica en un importante número de juicios, decisiones y acciones. En este sentido, contrastan decisiones que denominan de arriba hacia abajo (por influencia de un pasado - que puede ser nostálgico y positivo o traumático, aversivo y negativo; incluso, preciso o distorsionado- o por anticipación de metas, ponderación de relaciones entre medios y fines, de los componentes deseados y de los impedimentos y desafíos) con aquellas de abajo hacia arriba en la medida en que reciben influencia de las cualidades sensoriales, biológicas y sociales de los elementos relevantes del presente.

Cuando se desarrolla una tendencia habitual a enfatizar uno de estos tres marcos temporales, se conforma un "sesgo" cognitivo hacia el pasado, el presente o el futuro, una variable entre las diferencias individuales, característica y predictiva de las elecciones y respuestas de una persona. Se trata de una diferencia individual relativamente estable, proveniente de factores aprendidos, entre los que destacan la cultura, la familia, la educación, la religión y el nivel socioeconómico.

Para evaluar la perspectiva temporal, se emplea el Zimbardo Time Perspective Inventory-ZTPI (Zimbardo y Boyd, 1999), compuesto por 56 proposiciones sobre creencias, preferencias y valores respecto de experiencias

1 Carelli, Wiberg y Wiberg (2011, p. 220) lo caracterizan como "semi-consciente". 
temporalmente delimitadas. El inventario presenta 5 opciones de respuesta (desde "No me describe en absoluto" hasta "Me describe totalmente") y evalúa 5 dimensiones:

1. Pasado negativo: una actitud pesimista y visión aversiva del pasado (ejemplo de ítem del zTPI: "Es difícil olvidar imágenes desagradables de mi infancia"), que se puede asociar — pero no necesariamentea eventos traumáticos o resentimientos. Esto es, por la naturaleza reconstructiva del pasado, estas actitudes negativas pueden deberse a experiencias reales, a una reconstrucción negativa de eventos benignos o a una combinación de ambas.

2. Presente hedonista: se asocia a vivir el momento, al deseo del placer espontáneo, a la búsqueda de la gratificación inmediata con mínima consideración de los riesgos o las consecuencias de las acciones (por ejemplo, "Me arriesgo para poner excitación en mi vida").

3. Futuro: se caracteriza por la actitud de fijarse metas específicas de largo aliento, por la planificación de acciones futuras, la anticipación constante de consecuencias y recompensas, un trabajo sostenido hacia dichas metas (por ejemplo "Cuando quiero conseguir algo, me fijo unas metas y considero los medios para poder conseguirlas"), a expensas del disfrute presente o la capacidad de aplazar las gratificaciones.

4. Pasado positivo: refleja una actitud cálida, sentimental y usualmente nostálgica hacia el pasado, que enfatiza la preservación de las relaciones con la familia y los amigos (por ejemplo, "Las imágenes, sonidos y olores de la infancia traen recuerdos maravillosos").

5. Presente fatalista: se define por una actitud de falta de esperanza en el futuro, por la creencia de que la propia vida está determinada por fuerzas incontrolables (por ejemplo "No tiene sentido preocuparme por el futuro ya que, de todos modos, no puedo hacer nada").

En la literatura se advierte un amplio consenso que valida transculturalmente las cinco dimensiones que distinguieran los autores; por ejemplo, las investigaciones de Apostolidis y Fieulaine (2004) con estudiantes franceses; de Díaz-Morales (2006a y 2006b) con una población española; de Milfont, Andrade, Belo y Pessoa (2008) con alumnos brasileños, o el trabajo de Sircova et al. (2014) en países de los cinco continentes, con una varianza explicada promedio de $35,05 \%$, en el que se concluye:

Nuestros resultados muestran que las cinco orientaciones temporales evaluadas mediante el ZTPI Son invariantes a través de veinticuatro países con diversas tradiciones culturales y a través de muchas adaptaciones lingüísticas [...]. En consecuencia, podemos recomendar fuertemente las distintas versiones del ZTPI como un "estándar de oro" 
para la investigación futura de la perspectiva temporal. (Sircova et al., 2014, p. 8).

La profusa aplicación del zTPı ha mostrado que la orientación del estudiante a un pasado negativo o al presente (tanto hedonista como fatalista) se asocia con bajos niveles de autoestima y autoeficacia, previsión de metas a corto plazo, procrastinación (Ferrari y Díaz-Morales, 2007), e insuficientes estrategias autorregulatorias, lo que se traduce en fracaso académico (De Bilde et al., 2011; Horstmanshof y Zimitat, 2007). Por su parte, el alumno que confía en sus habilidades (pasado positivo) y en que su esfuerzo produce resultados (bajo presente fatalista) es más proclive a trabajar por el logro de sus metas (Horstmanshof y Zimitat, 2007). Finalmente, la orientación al futuro, como señalamos, aparece como una PT más constructiva, optimista, que preserva un funcionamiento positivo y que suele conducir al logro académico y profesional. No obstante, una fijación desmedida en metas futuras podría comprometer la espontaneidad y dar paso a una conducta poco flexible y una habilidad deficiente para disfrutar el presente (Boniwell y Zimbardo, 2003). Por ello, Zimbardo y Boyd postulan que la Pт ideal es "balanceada" (1999, p. 1285), entendida como la habilidad mental para flexibilizar la PT en función de las demandas de la tarea, aspectos de la situación y la evaluación de los recursos personales: el foco en el futuro proporciona a la persona "alas para ascender a nuevas cumbres de logro", en el pasado positivo "arraiga sus raíces [sic] en la tradición y fundamenta el sentimiento de identidad personal" y en el presente hedonista "alimenta la vida cotidiana" mediante la alegría y la disposición a actividades lúdicas.

\section{Metodología}

Se implementó un diseño ecológico seccional cuantitativo, de profundidad exploratoria, descriptiva y correlacional.

\section{Participantes}

La muestra ( $N=616$ ) estuvo conformada por estudiantes universitarios de primero a cuarto año de algunas carreras (Letras, Geografía, Inglés y Ciencias de la educación) de la Facultad de Filosofía y Letras ( $\mathrm{N}=328$ ) y de Ingeniería Agronómica $(\mathrm{N}=288)$ de la Facultad de Ciencias Agrarias. Ambas instituciones pertenecen a la Universidad Nacional de Cuyo (Mendoza, Argentina).

La muestra (no probabilística y por conveniencia) se seleccionó en función de los objetivos planteados: alumnos de primero a cuarto año y de diversas carreras, a fin de propender a mayor variabilidad en patrones de aprendizaje y patrones temporales. 


\section{Procedimiento e instrumentos}

En situación regular de clase, en dos sesiones, en presencia del profesor, se les explicó a los alumnos el propósito general de la investigación, se les indicó que la participación era voluntaria y que podían interrumpirla en cualquier momento del proceso. Se obtuvo su consentimiento y se les informó el procedimiento de respuesta.

En primer lugar, se aplicó la versión española del ıLs de Jan Vermunt (1998), traducida y validada por Martínez-Fernández et al. (2009). ${ }^{2}$ Luego se efectuó, un análisis factorial exploratorio ${ }^{3}$ en cuatro factores con rotación Quartimax ( $\left.\mathrm{KMO}=0,808 ; X^{2}=3336,577, \mathrm{p}<0,01\right)$, el cual retiene las 20 subescalas, con índices de confiabilidad entre 0,40 (en Interés personal) y 0,85 (Conocimiento como estímulo del docente), cercanos a los obtenidos en una investigación con estudiantes universitarios españoles y latinoamericanos (Martínez-Fernández y Vermunt, 2015), cuyos extremos también corresponden a dichos componentes, con índices de 0,34 y 0,88, respectivamente. Sin embargo, resultan inferiores a los del estudio original (Vermunt, 1994) en el cual los índices oscilan entre 0,48 (Regulación externa de los procesos) y 0,89 (Conocimiento como cooperación entre pares). Por otra parte, todos los indicadores son discriminativos en la confrontación de grupos extremos mediante la t de Student.

En la segunda sesión se administró la adaptación al español del ZTPI de Zimbardo y Boyd desarrollada por Díaz-Morales (2006a y 2006b). Un análisis factorial exploratorio en cuatro factores con rotación Promax $\left(\mathrm{KMO}=0,867 ; \mathrm{X}^{2}=12667,02, \mathrm{p}=0,000\right)$ aconseja retener 33 ítems, dado que algunos indicadores no cargan en factor alguno o en la estructura original: 6 de 10 en Pasado negativo $(\alpha=0,61), 8$ de 15 en Presente hedonista $(\alpha=0,66), 8$ de 13 en Futuro $(a=0,71), 6$ de 9 en Pasado positivo ( $a=0,67)$ y 5 de 9 en Presente fatalista $(a=0,61)$. Los coeficientes de confiabilidad hallados (entre 0,61 y 0,71 ) se acercan más a la investigación de Sircova et al. (2014), en la cual oscilan entre 0,60 y 0,77, que a la versión original (Zimbardo y Boyd, 1999), que obtiene índices entre 0,74 y 0,82. Nuevamente, la totalidad de los ítems resulta discriminativa.

Para el análisis de los datos, mediante el programa spss 22.0, se aplicaron los siguientes estadísticos: descriptivos, prueba de significación de la diferencia de proporciones, chi cuadrado y conglomerados de $\mathrm{k}$ medias.

2 Miembros de un equipo internacional denominado Grupo de Investigación, Docencia e Intervención en Patrones de Aprendizaje y Formación Investigadora en la Universidad G_Pafiu, liderado por J. Reinaldo Martínez-Fernández desde la Universidad Autónoma de Barcelona, del cual forman parte las autoras del presente artículo desde 2011.

3 Se presenta una síntesis apretada de las cualidades psicométricas de los instrumentos para no distraer del propósito del presente trabajo. 


\section{Resultados}

Ordenaremos la presentación de los resultados siguiendo un esquema en cuatro partes: patrones de aprendizaje, patrones temporales, variables personales y contextuales, y vinculación entre patrones de aprendizaje y temporales.

\section{Patrones de aprendizaje}

Mediante la adaptación del procedimiento descrito por Mumbardó y Martínez-Fernández (en comunicación personal) para la adscripción de los patrones a los casos, ${ }^{4}$ se halla la siguiente distribución en la muestra (tabla 1), que deja ver diferencias de proporciones con significatividad estadística entre UD y MD $(z=3,065 ; p<0,01)$, RD y AD $(z=2,635 ; p<0,01)$ y UD y $\operatorname{AD}(z=4,623 ; p<0,01)$.

Tabla 1.

Frecuencia de patrones de aprendizaje

\begin{tabular}{lcc}
\hline \multicolumn{1}{c}{ Patrones de aprendizaje } & Frecuencia & $\%$ \\
\hline Dirigido al significado (MD) & 140 & 22,7 \\
\hline Orientado a la aplicación (AD) & 118 & 19,2 \\
\hline Orientado a la reproducción (RD) & 160 & 26,0 \\
\hline No orientado (UD) & 198 & 32,1 \\
\hline Total & 616 & 100,0 \\
\hline
\end{tabular}

Fuente: elaboración propia.

Respecto de las variables personales, ni el género ni la edad establecen diferencias estadísticamente significativas $\left(X^{2}=2,117, g l=3, p=0,549\right.$; $X^{2}=14,724, g l=9, p=0,099$; respectivamente). En relación con las variables contextuales, los porcentajes por carrera se presentan en la tabla 2.

Tabla 2.

Tabla de contingencia patrón de aprendizaje por carreras

\begin{tabular}{lcccccc}
\hline \multirow{2}{*}{$\begin{array}{c}\text { Patrón de } \\
\text { aprendizaje }\end{array}$} & \multicolumn{6}{c}{ Carreras } \\
\cline { 2 - 7 } & Ingeniería & Educación & $\begin{array}{c}\text { Geografial } \\
\text { Letras }\end{array}$ & Inglés & Total \\
\hline $\begin{array}{l}\text { Dirigido al } \\
\text { significado }\end{array}$ & Recuento & 79 & 30 & 20 & 11 & 140 \\
\cline { 2 - 7 } & $\%$ & 24 & 29 & 25,5 & 10 & 23 \\
\hline $\begin{array}{l}\text { Dirigido a la } \\
\text { aplicación }\end{array}$ & Recuento & 83 & 19 & 11 & 5 & 118 \\
\cline { 2 - 7 } & $\%$ & 25 & 19 & 14 & 5 & 19 \\
\hline $\begin{array}{l}\text { Dirigido a la } \\
\text { reproducción }\end{array}$ & Recuento & 72 & 21 & 24 & 43 & 160 \\
\cline { 2 - 7 } & $\%$ & 22 & 21 & 31 & 40 & 26 \\
\hline
\end{tabular}

4 Se puede acceder a este procedimiento solicitándolo a las autoras. 


\begin{tabular}{lcccccc}
\hline \multirow{2}{*}{$\begin{array}{c}\text { Patrón de } \\
\text { aprendizaje }\end{array}$} & \multicolumn{6}{c}{ Carreras } \\
\cline { 2 - 7 } & Ingeniería & Educación & $\begin{array}{c}\text { Geografia/ } \\
\text { Letras }\end{array}$ & Inglés & Total \\
\hline \multirow{2}{*}{ No orientado } & Recuento & 94 & 32 & 23 & 49 & 198 \\
\cline { 2 - 7 } & $\%$ & 29 & 31 & 29.5 & 45 & 32 \\
\hline Total & Recuento & 328 & 102 & 78 & 108 & 616 \\
\hline
\end{tabular}

Fuente: elaboración propia.

Las diferencias estadísticamente significativas $\left(X^{2}=48,610, \mathrm{gl}=9\right.$, $p=0,000$ ) más sobresalientes son: los alumnos de Ciencias de la Educación manifiestan una frecuencia algo mayor que los de las restantes carreras en patrón dirigido al significado; los de Ingeniería superan a las estudiantes de Ciencias de la Educación, Geografía/Letras e Inglés en patrón orientado a la aplicación; en los estudiantes de Inglés prevalecen el patrón reproductivo y el no orientado (en ambos, con los porcentajes mayores de la muestra).

En la tabla 3 se presentan los resultados en relación con el curso. Se hallan, entonces, diferencias estadísticamente significativas $\left(X^{2}=53,347\right.$, $\mathrm{gl}=9, \mathrm{p}=0,000$ ) en la distribución de los patrones de aprendizaje por curso: mientras en los alumnos de primero y segundo el porcentaje mayor corresponde a los patrones no orientado y orientado a la reproducción, respectivamente; más del $50 \%$ de los de cuarto se concentran en el dirigido al significado. En cambio, los estudiantes de tercero manifiestan una ubicación más propia de los cursos inferiores, ya que el $82 \%$ se concentra entre el patrón reproductivo y el no orientado. Podríamos decir que a medida que se avanza en la carrera hay una variación sistemática de los patrones de aprendizaje en el sentido de que aumentan los patrones MD/AD y disminuyen los RD/UD. No se observa lo mismo en los estudiantes de tercer año.

\section{Tabla 3.}

Tabla de contingencia patrón de aprendizaje por curso (año que cursa en la carrera)

\begin{tabular}{|c|c|c|c|c|c|c|}
\hline \multirow{2}{*}{$\begin{array}{c}\text { Patrón de } \\
\text { aprendizaje }\end{array}$} & & \multicolumn{5}{|c|}{ Curso } \\
\hline & & $1 .^{\circ}$ & $2 .^{\circ}$ & $3 .^{\circ}$ & $4 .^{\circ}$ & Total \\
\hline \multirow{2}{*}{$\begin{array}{l}\text { Dirigido al } \\
\text { significado }\end{array}$} & Recuento & 103 & 12 & 1 & 24 & 140 \\
\hline & $\%$ & 21 & 24,5 & 4 & 54,5 & 23 \\
\hline \multirow{2}{*}{$\begin{array}{l}\text { Dirigido a la } \\
\text { aplicación }\end{array}$} & Recuento & 100 & 8 & 1 & 9 & 118 \\
\hline & $\%$ & 20 & 16 & 4 & 20,5 & 19 \\
\hline \multirow{2}{*}{$\begin{array}{l}\text { Dirigido a la } \\
\text { reproducción }\end{array}$} & Recuento & 121 & 20 & 14 & 5 & 160 \\
\hline & $\%$ & 24 & 40,5 & 54 & 11 & 26 \\
\hline \multirow{2}{*}{ No orientado } & Recuento & 173 & 9 & 10 & 6 & 198 \\
\hline & $\%$ & 35 & 18 & 38 & 14 & 32 \\
\hline Total & Recuento & 497 & 49 & 26 & 44 & 616 \\
\hline
\end{tabular}

Fuente: elaboración propia. 


\section{Patrones temporales}

El patrón temporal se conforma mediante un análisis de conglomerados de $k$ medias con las cinco escalas del zTPI. ${ }^{5}$

El primer conglomerado corresponde al patrón temporal orientado al futuro. Del mismo modo que investigaciones previas (Díaz-Morales, 2006a y 2006b; Horstmanshof y Zimitat, 2007; Zimbardo y Boyd, 1999), se caracteriza también por la media inferior de la distribución en Presente fatalista; no obstante, manifiesta una conformación idiosincrática, diferente de aquellas investigaciones, por cuanto evidencia un valor medio alto en Pasado negativo.

El segundo remite claramente al patrón orientado al pasado, ya que presenta las medias más altas de la distribución en Pasado negativo y Pasado positivo.

El tercero —que hemos denominado "interrumpido" siguiendo a Gutiérrez-Braojos et al. (2014) — se distingue por las medias más bajas en cuatro de las dimensiones, lo que indica una ausencia de orientación temporal (tabla 4). Por otra parte, es el único que muestra una diferencia de proporción con significatividad estadística $(z=2.196 ; p<0,05)$ en comparación con el de menor porcentaje (el orientado al pasado).

\section{Tabla 4.}

Centros de los conglomerados finales en las escalas del ZPTI

\begin{tabular}{lccc}
\hline & \multicolumn{3}{c}{ Conglomerados } \\
\hline & $\mathbf{1}$ & $\mathbf{2}$ & $\mathbf{3}$ \\
\hline Pasado negativo & $7,3(34,6 \%)$ & $N=179(29 \%)$ & $N=224(36,4 \%)$ \\
\hline Presente hedonista & 6,41 & 8,06 & 5,78 \\
\hline Futuro & 7,52 & 6,09 & 4,71 \\
\hline Pasado positivo & 5,44 & 5,53 & 5,34 \\
\hline Presente fatalista & 4,20 & 7,02 & 4,84 \\
\hline Fun & & 6,10 & 5,20 \\
\hline
\end{tabular}

Fuente: elaboración propia.

5 Se les solicitan tres conglomerados a partir del número que se conforma en un análisis previo en dos fases, en el que promedio con aplazos (esto es, que incluye también las calificaciones de los exámenes desaprobados) se ingresa como variable numérica, por su relevancia en la investigación que se reseña. Como variable de entrada para calcular los clusters, se utilizan los resultados obtenidos de las cinco escalas del zTPI. 


\section{Variables personales y contextuales}

La asociación con el género no es estadísticamente significativa $\left(X^{2}=4,112, g l=2, p=0,128\right) .{ }^{6}$ Es un resultado que no sorprende, por cuanto los estudios constreñidos a la pTF muestran resultados no concluyentes en relación con esta variable (Greene y DeBacker, 2004) o ningún efecto con significatividad estadística (Peetsma, 2000). Con respecto a la edad, en cambio, sí hay una asociación significativa $\left(X^{2}=85,100, g l=6, p=0,000\right)$.

Son los estudiantes más jóvenes quienes muestran los porcentajes más altos en orientación al futuro y al pasado, al tiempo que en los más grandes prima el patrón interrumpido (tabla 5).

\section{Tabla 5.}

Tabla de contingencia patrón temporal por edad

\begin{tabular}{|c|c|c|c|c|c|c|}
\hline \multirow{2}{*}{$\begin{array}{l}\text { Patrón } \\
\text { temporal }\end{array}$} & \multicolumn{6}{|c|}{ Edad } \\
\hline & & 17 años & 18 años & $\begin{array}{c}19-20 \\
\text { años }\end{array}$ & $\begin{array}{l}21 \text { años } \\
\text { o más }\end{array}$ & Total \\
\hline \multirow{2}{*}{ Al futuro } & Recuento & 45 & 66 & 62 & 40 & 213 \\
\hline & $\%$ & 40 & 38 & 37 & 25 & 34,5 \\
\hline \multirow{2}{*}{ Al pasado } & Recuento & 45 & 62 & 55 & 17 & 179 \\
\hline & $\%$ & 40 & 36 & 32 & 10 & 29 \\
\hline \multirow{2}{*}{ Interrumpido } & Recuento & 22 & 45 & 52 & 105 & 224 \\
\hline & $\%$ & 20 & 26 & 31 & 65 & 36,5 \\
\hline Total & Recuento & 112 & 173 & 169 & 162 & 616 \\
\hline
\end{tabular}

Fuente: elaboración propia.

Respecto de la primera variable contextual, los resultados se presentan en la tabla 6. La significatividad estadística de las relaciones $\left(X^{2}=285,354, \mathrm{gl}=6, \mathrm{p}=0,000\right)$ procede de la mayor orientación al futuro de los estudiantes de Ciencias de la Educación y de Geografía/Letras, en comparación con la prevalencia cierta del patrón interrumpido en los alumnos de Inglés y la mayor concentración en el pasado en el caso de los estudiantes de Ingeniería.

6 Se halla que las interacciones entre género, edad, familia, estatus socioeconómico y cultura son bastante imbrincadas (quite involved), lo que revela que el constructo es complejo, profundamente vinculado con el ambiente sociocultural y familiar (Greene y DeBacker, 2004). 
Tabla 6.

Tabla de contingencia patrón temporal por carrera

\begin{tabular}{ccccccc}
\hline \multirow{2}{*}{$\begin{array}{c}\text { Patrón } \\
\text { temporal }\end{array}$} & \multicolumn{6}{c}{ Carreras } \\
\cline { 2 - 7 } & Ingeniería & Educación & $\begin{array}{c}\text { Geografia/ } \\
\text { Letras }\end{array}$ & Inglés & Total \\
\hline \multirow{2}{*}{ Al futuro } & Recuento & 103 & 57 & 47 & 6 & 213 \\
\cline { 2 - 7 } & $\%$ & 31 & 56 & 60 & 5,5 & 34,5 \\
\hline \multirow{2}{*}{ Al pasado } & Recuento & 163 & 7 & 8 & 1 & 179 \\
\cline { 2 - 7 } & $\%$ & 50 & 7 & 10 & 1 & 29 \\
\hline \multirow{2}{*}{ Interrumpido } & Recuento & 62 & 38 & 23 & 101 & 224 \\
\cline { 2 - 7 } & Recuento & 328 & 102 & 78 & 108 & 616 \\
\hline Total & 19 & 37 & 30 & 93,5 & 36,5 \\
\hline
\end{tabular}

Fuente: elaboración propia.

En cuanto a los resultados en función del curso (tabla 7 ) se presentan algunas discrepancias estadísticamente significativas $\left(X^{2}=63.083, \mathrm{gl}=6\right.$, $p=0,000$ ) porque, mientras que los alumnos de primero se distribuyen con cierta uniformidad entre los tres patrones, los de segundo se ubican en los polos (al futuro e interrumpido), los de tercero y cuarto tampoco muestran orientación al pasado pero se concentran en el interrumpido. Por su parte, los de cuarto también evidencian un porcentaje medio de orientación al futuro.

Tabla 7.

Tabla de contingencia patrón temporal por curso

\begin{tabular}{|c|c|c|c|c|c|c|}
\hline \multirow{2}{*}{$\begin{array}{l}\text { Patrón } \\
\text { temporal }\end{array}$} & & \multicolumn{5}{|c|}{ Curso } \\
\hline & & $1 .^{\circ}$ & $2 .^{\circ}$ & $3 .^{\circ}$ & $4 .^{\circ}$ & Total \\
\hline \multirow{2}{*}{ Al futuro } & Recuento & 171 & 24 & 3 & 15 & 213 \\
\hline & $\%$ & 34,4 & 49 & 11,5 & 34 & 34,5 \\
\hline \multirow{2}{*}{ Al pasado } & Recuento & 172 & 4 & 1 & 2 & 179 \\
\hline & $\%$ & 34,6 & 8 & 4 & 4,5 & 29 \\
\hline \multirow{2}{*}{ Interrumpido } & Recuento & 154 & 21 & 22 & 27 & 224 \\
\hline & $\%$ & 31 & 43 & 84,5 & 61,5 & 36,5 \\
\hline Total & Recuento & 497 & 49 & 26 & 44 & 616 \\
\hline
\end{tabular}

Fuente: elaboración propia.

\section{Vinculación entre patrones de aprendizaje y temporales}

Respecto de la interacción entre patrones de aprendizaje y temporales (tabla 8 ), se encuentra que esta es estadísticamente significativa $\left(X^{2}=90,435, g l=6, p=0,000\right)$ por la prevalencia de la orientación al 
futuro en los alumnos dirigidos al significado, y del patrón temporal interrumpido en los no orientados. Esto es, los resultados de mayor interés para la vinculación que se tiene por hipótesis son, por un lado, que los dos porcentajes más altos corresponden al patrón orientado al significado y al futuro (37\%), y al no orientado e interrumpido (51\%); por otro, que los patrones de aprendizaje MD y AD evidencian los índices más bajos en su asociación con el patrón temporal interrumpido (13\% en el primer caso y $10 \%$ en el segundo), mientras que los estudiantes dirigidos a la reproducción se distribuyen con cierta uniformidad entre los tres patrones temporales (figura 1).

\section{Tabla 8.}

Contingencia patrón de aprendizaje por patrón temporal

\begin{tabular}{|c|c|c|c|c|c|}
\hline \multirow[b]{2}{*}{ Patrón de aprendizaje } & \multicolumn{5}{|c|}{ Patrón Temporal } \\
\hline & & $\begin{array}{l}\text { Orientado } \\
\text { al futuro }\end{array}$ & $\begin{array}{l}\text { Orientado } \\
\text { al pasado }\end{array}$ & Interrumpido & Total \\
\hline \multirow{2}{*}{ Dirigido al significado } & Recuento & 79 & 33 & 28 & 140 \\
\hline & $\%$ & 37 & 18.5 & 13 & 23 \\
\hline \multirow{2}{*}{ Dirigido a la aplicación } & Recuento & 46 & 49 & 23 & 118 \\
\hline & $\%$ & 22 & 27.5 & 10 & 19 \\
\hline \multirow{2}{*}{$\begin{array}{l}\text { Dirigido a la } \\
\text { reproducción }\end{array}$} & Recuento & 50 & 52 & 58 & 160 \\
\hline & $\%$ & 23 & 29 & 26 & 26 \\
\hline \multirow{2}{*}{ No orientado } & Recuento & 38 & 45 & 115 & 198 \\
\hline & $\%$ & 18 & 25 & 51 & 32 \\
\hline Total & Recuento & 213 & 179 & 224 & 616 \\
\hline
\end{tabular}

Fuente: elaboración propia.

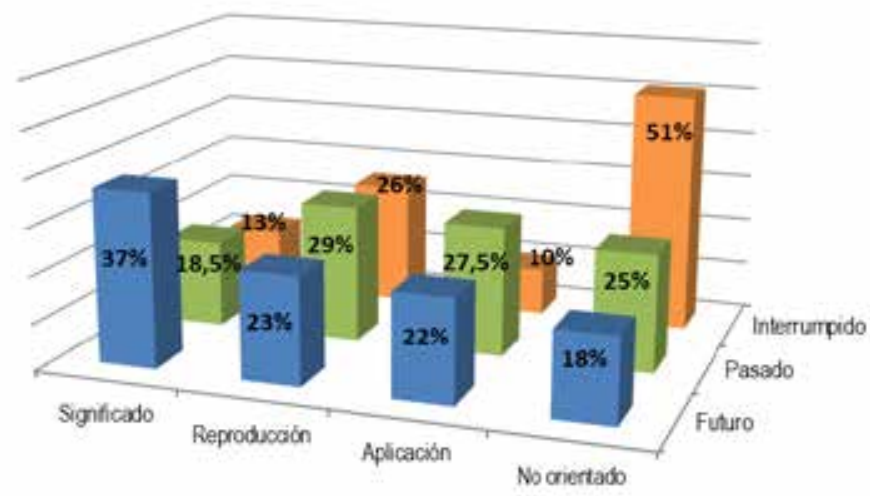

Figura I. Patrón de aprendizaje por patrón temporal

Fuente: elaboración propia. 


\section{Discusión y conclusiones}

La presente investigación se propuso analizar, en estudiantes de algunas carreras de la Universidad Nacional de Cuyo (Mendoza, Argentina): 1) la conformación de patrones de aprendizaje (Vermunt, 1996, 1998, 2005) y patrones temporales (Gutiérrez-Braojos et al., 2014; Zimbardo y Boyd, 1999); 2) la relación de dichos patrones con variables personales (edad y género) y contextuales (carrera y año que cursa); y 3) la vinculación existente entre los patrones de aprendizaje y los temporales.

Tal como lo propone Vermunt (1998), en la muestra argentina se identifica una estructura de cuatro patrones de aprendizaje, con prevalencia estadísticamente significativa de los menos deseados: UD y RD, en ese orden.

Por otra parte, se conforman tres patrones temporales: orientado al futuro, orientado al pasado e interrumpido; el último muestra mayor porcentaje que los otros dos. Entonces, también en el ámbito de la PT prima el tipo menos deseado.

Respecto de la combinación entre patrones de aprendizaje y patrones temporales, se halla que prevalecen el orientado al significado y al futuro y el no orientado e interrumpido.

El primero caracteriza a los estudiantes que emplean estrategias de procesamiento profundo (relación-estructuración y pensamiento crítico) y de regulación de los contenidos, procesos y resultados del aprendizaje; muestran un interés personal en el tema que se aborda y sus modelos mentales implican la concepción del conocimiento como construcción del significado. Se fijan metas específicas de largo aliento, planifican acciones futuras y se anticipan de manera constante a las consecuencias y recompensas.

El patrón no orientado e interrumpido se distingue por estudiantes que, para llevar a cabo las actividades de aprendizaje, necesitan estímulos y apoyos por parte de sus docentes y compañeros, evidencian una orientación anclada en el presente con una tendencia hedonista y una interrupción en la construcción de representaciones mentales futuras.

Por su parte, los alumnos que se orientan a la reproducción (esto es, con una fuerte orientación externa, cuya principal preocupación es repetir el contenido para aprobar los exámenes) se distribuyen proporcionalmente en las tres orientaciones temporales, lo que permitiría anticipar (con la debida cautela por las características de la muestra) que el patrón de aprendizaje es su rasgo distintivo. En cambio, los estudiantes orientados a la aplicación (aquellos que se caracterizan por el procesamiento concreto y el empleo de lo aprendido en situaciones actuales) se concentran, en porcentajes similares, en la orientación al futuro y al pasado. 
En cuanto a la relación existente entre los patrones y los factores personales y contextuales, se hallan diferencias significativas por edad (variable personal) solo en el caso de los patrones temporales. Los estudiantes más jóvenes muestran los porcentajes más altos en orientación al futuro y al pasado, al tiempo que en los más grandes prima el patrón interrumpido. Este resultado, que se contrapone a estudios previos en alumnos universitarios (Laureiro-Martínez et al., 2017), se puede explicar por la conformación del grupo de 21 años o más ya que se trata, en un porcentaje considerable, de estudiantes que están retrasados en su carrera. La otra variable personal (género), en contraste con la investigación previa, no arrojó diferencias significativas en combinación con los patrones de aprendizaje (Severiens y Dam, 1995) ni tampoco con los temporales (Brenlla, Willis y Germano, 2016; Greene y De Backer, 2004).

Respecto de las variables contextuales interesan las asociaciones — previsibles por la investigación previa (García-Ravidá, 2017; Martínez-Fernández y García-Ravidá, 2012) — con el patrón de aprendizaje. Desde la carrera, la primacía de MD en estudiantes de Ciencias sociales (específicamente, de Ciencias de la educación) y de AD en alumnos de Ingeniería; desde el curso, mientras que en los alumnos de primero y segundo el porcentaje mayor corresponde a los patrones UD y RD, respectivamente, más del $50 \%$ de los de cuarto se concentran en un patrón MD.

En síntesis, resulta de interés replicar esta investigación en una muestra diversificada de estudiantes argentinos (por ejemplo, de distintas regiones y de otras carreras) con el fin de contrastar si se verifican las regularidades halladas y de profundizar en sus razones. Especialmente, parece importante indagar la ocurrencia de los patrones combinados y su asociación con el desempeño académico en los distintos tramos de la trayectoria universitaria.

En el plano aplicado, la relevancia de identificar patrones orientados al significado y al futuro (MD y PTF), orientados a la reproducción y al pasado (RD y PTP) e interrumpidos (PTI) es que posibilita brindar lineamientos para loptimizar los procesos de aprendizaje. Por mencionar uno: diseñar ciertas actividades para los estudiantes en espacios comunes, a los que se les asignen diferentes roles en función de su perfil de aprendizaje y motivacional, a fin de que puedan mejorar los componentes del patrón de aprendizaje y, eventualmente, encaminarse hacia una PT más balanceada. Esta propuesta pedagógica está en línea con los resultados de estudios recientes (Beccaria, Kek, Huisjer, Rose, y Kimmis, 2014; Martínez-Fernández, García-Orriols y Galera Basachs, 2017; Martínez-Fernández, García-Ravidá, García-Orriols y Martí-Garbayo, 2018). 
Finalmente, y coincidiendo con la investigación de Gutiérrez-Braojos et al. (2014), es importante considerar para próximos estudios que el patrón temporal podría ser un factor explicativo, o encontrarse relacionado con el cambio cualitativo que sucede en los estudiantes cuando pasan de estar regulados externamente a autorregularse. En tal sentido, cobra vital relevancia el análisis de las fases de transición educativa.

\section{Referencias}

Adelabu, D. (2007). Time perspective and school membership as correlates to academic achievement among African American adolescents. Adolescence, 42(167), 525-538.

Aguilar-Rivera, M. del C. (2010). Los enfoques de aprendizaje en la universidad: un estudio de caso [en línea]. Revista de Psicología, 6(11), 67-86. Recuperado de http://bibliotecadigital.uca.edu.ar/repositorio/ revistas/enfoquesaprendizajeuniversidad-estudio-caso.pdf [Fecha de consulta: 10/ 10/2015].

Apostolidis, T. y Fieulaine, N. (2004). Validation francaise de l'echelle de temporalite. Revue Europeenne de Psychologie Appliquee, 54, 207 217. Dol:10.1016/j.erap.2004.03.001.

Bandura, A. (1997). Self-efficacy: the exercise of control. Nueva York: W. H. Freeman.

Beccaria, L. Kek, M., Huisjer, H., Rose, J. y Kimmis, L. (2014). The interrelationships between student approaches to learning and group work. Nurse Education Today, 34, 1094-1103.

Boniwell, I. y Zimbardo, P. (2003). Time to find the right balance. The Psychologist, 16, 129-131.

Brenlla, M. E., Willis, B. y Germano, G. (2016). Estimación del tiempo y perspectiva temporal en distintas etapas de la adultez. Investigaciones en Psicología, 21(1), 27-34.

Carelli, M. G., Wiberg, B. y Wiberg, M. (2011). Development and construct validation of the Swedish Zimbardo Time Perspective Inventory. European Journal of Psychological Assessment, 27(4), 220-227. DOI: 10.1027/1015-5759/a000076.

De Bilde, J., Vansteenkiste, M. y Lens, W. (2011). Understanding the association between future time perspective and self-regulated learning through the lens of self-determination theory. Learning and Instruction, 21, 332-344. DOI: 10.1016/j.learninstruc.2010.03.002.

Díaz-Morales, J. F. (2006a). Factorial structure and reliability of Zimbardo Time Perspective Inventory. Psichotema, 18, 565-571.

Díaz-Morales, J. F. (2006b). Future time perspective across life-span: two models of measurement. En: Z. Uchnast (ed.), Psychology of time. Theoretical and empirical approaches (pp. 65-77). Lublin: Wydawnictwo KUL. 
Entwistle, N. J., McCune, V. y Walker, P. (2001). Conceptions, styles and approaches within higher education: Analytical abstractions and everyday experience. En R. J. Sternberg y L. F. Zhang (eds.), Perspectives on thinking, learning and cognitive styles (pp. 103-136). Mahwah, NJ: Erlbaum.

Ferrari, J. y Diaz-Morales, J. (2007). Procrastination: Different time orientations reflect different motives. Journal of Research in Personality, 41, 707-714. DOI: 10.1016/j.jrp.2006.06.006.

García-Fuentes, C., Muñoz-Cantero J. y Abalde-Paz, E. (2002). Universitarios y profesionales, diagnóstico de estilos de aprendizaje. Revista de Investigación Educativa, 20(2), 339-356.

García-Ravidá, L. (2017) Patrones de aprendizaje en universitarios latinoamericanos: dimensión cultural e implicaciones educativas (tesis doctoral). Universidad Autónoma de Barcelona, Barcelona, España.

Green, B. y DeBacker, T. (2004). Gender and orientations toward the future: Links to Motivation. Revista de Investigación Educativa, 16(2), 303-337.

Gutiérrez-Braojos, C., Salmerón-Pérez, H. y Muñoz-Cantero, J. (2014). El efecto modulador de los patrones temporales sobre el logro en el aprendizaje autorregulado. Revista de Psicodidáctica, 19(2), 267 287. DOI: 10.1387/RevPsicodidact.10066.

Horstmanshof, L. y Zimitat, C. (2007). Future time orientation predicts academic engagement among first-year university students. British Journal of Educational Psychology, 77(3), 703-718. DOI: 10.1348/000709906x160778.

Keefe, J. W. (1985). Assessment of learning style variables. The NASSP task force model. Theory into Practice, 24(2), 138-144.

Laureiro-Martínez, D., Trujillo, C. A. y Unda, J. (2017). Time perspective and age: A review of age associated differences. Frontiers in Psychology, 8(101), 1-8. Dol:10.3389/fpsyg.2017.00101.

Marín Gracia, M. (2002). La investigación sobre diagnóstico de los estilos de aprendizaje en la enseñanza superior. Revista de Investigación Educativa, 20(2), 303-337.

Martínez-Fernández, J. R. et al. (2009). ıs Inventory of learning styles (en español). Barcelona: Universitat Autònoma de Barcelona. Recuperado de http://grupsderecerca.uab.cat/pafiu/sites/grupsderecerca.uab. cat.pafiu/files/ıs_Castellano.pdf.

Martínez-Fernández, J. R., García-Orriols, J. y Galera-Bassachs, A. (2017). Patrones de aprendizaje en educación primaria. Identificación y acciones formativas inclusivas. En Manual de Educación Primaria. Orientaciones y recursos. 6-12 años. Barcelona: Wolters Kluwer. 
Martínez-Fernández, J. R., García-Ravidá, L., García Orriols, J. y Martí Garbayo, L. (2018). Desarrollo personal y aprendizaje: desafíos a la escuela desde una mirada longitudinal a los patrones de aprendizaje. Contextos de Educación, 25, 54-66.

Martínez-Fernández, J. R. y García-Ravidá, L. (2012). Patrones de aprendizaje en estudiantes universitarios del Máster en Educación Secundaria: variables personales y contextuales relacionadas. Revista de Currículum y Formación del Profesorado, 16(1), 166-182.

Martínez-Fernández, J. R. y Vermunt, J. (2015). A cross-cultural analysis of the patterns of learning and academic performance of Spanish and Latin-American undergraduates. Studies in Higher Education, 40(2), 278-295. DOI: 10.1080/03075079.2013.823934.

Mclnerney, D. M. (2004). A discussion of future time perspective. Educational Psychology Review, 16(2), 141-151. DOI: 10.1023/B:EDPR.0000026610.18125.a3.

Milfont, T. L., Andrade, P. R., Belo, R. P. y Pesoa, V. S. (2008). Testing Zimbardo Time Perspective Inventory in a Brazilian sample. Interamerican Journal of Psychology, 42, 1-10.

Miller, R. y Brickman, S. (2004). A model of future-oriented motivation and self-regulation. Educational Psychology Review, 16(1), 9-33.

Nurmi, J. (1989). Development of orientation to the future during early adolescence: A four year longitudinal study and two cross-sectional comparisons. International Journal of Psychology, 24, 195-214.

Nuttin, J. (1985). Future time perspective and motivation: Theory and research method. Hillsdale, NJ: Erlbaum.

Peetsma, T. (2000). Future time perspective as a predictor of school investment. Scandinavian Journal of Educational Research, 44(2), 177-192,

Peetsma, T., Hascher, T., van der Veen, I. y Roede, E. (2005). Relations between adolescents' self-evaluations, time perspectives, motivation for school and their achievement in different countries and at different ages. European Journal of Psychology of Education, 20, 209-225. DOI: $10.1007 / \mathrm{BF} 03173553$.

Severiens, S. y Dam, G. T. (1995). Gender and gender identity differences in learning styles. Educational Psychology, 17(1-2), 79-93. Dol: 10.1080/0144341970170105.

Shell, D. F. y Husman, J. (2001). The multivariate dimensionality of personal control and future time perspective in achievement and studying. Contemporary Educational Psychology, 26, 481-506. DOI: 10.1006/ ceps.2000.1073.

Simons, J., Vansteenkiste, M., Lens, W. y Lacante, M. (2004). Placing motivation and future time perspective theory in a temporal pers-

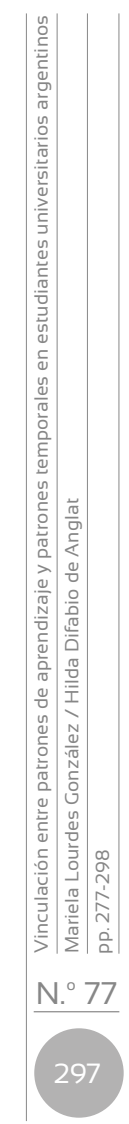


pective. Educational Psychology Review, 16(2), 121-139. DOI: 1040-726X/04/0600-0121/0.

Sircova, A., Van De Vijver, F., Osin, E., Milfont, T. L., Fieulaine, N., Kislali, A., Zimbardo, P. G. y 54 miembros del International Time Perspective Research Project (2014). A global look at time: A 24-country study of the equivalence of the Zimbardo Time Perspective Inventory. Sage Open, January-March, 1-12. DOI: 10.1177/2158244013515686.

Suddendorf, T. y Busby, J. (2005). Making decisions with the future in mind: Developmental and comparative identification of mental time travel. Learning Motivation, 36, 110-125. DOI: 10.1016/j.Imot.2005.02.010.

Vermunt, J. (1994). Inventory of Learning Styles in higher education: Scoring key. Tilburg University: Department of Educational Psychology.

Vermunt, J. (1996). Meta-cognitive, cognitive and affective aspects of learning styles and strategies: A phenomenographic analysis. Higher Education, 31(1), 25-50.

Vermunt, J. (1998). The regulation of constructive learning processes. British Journal of Educational Psychology, 68(2), 149-171. DOI: 10.1111/ j.2044-8279.1998.tb01281.

Vermunt, J. (2005). Relations between student learning patterns, personal and contextual factors and academic performance. Higher Education, 49(3), 205-234.

Worrell, F. C. y Mello, Z. R. (2007). The reliability and validity of Zimbardo Time Perspective Inventory scores in academically talented adolescents. Educational \& Psychological Measurement, 67, 487-504. DOI: 10.1177/0013164406296985.

Zerbardast, A., Ali Besharat, M. y Hghighatgoo, M. (2011). The relationship between self-efficacy and time perspective in students. Procedia-Social and Behavioral Sciences, 30, 935-938. DOI: 10.1016/j. sbspro.2011.10.181.

Zimbardo, P. y Boyd, N. (1999). Putting time in perspective: A valid, reliable, individual-differences metric. Journal of Personality and Social Psychology, 17(6), 1271-1288. Dol: 10.1177/0961463x13487043.

Zimbardo, P. G. y Boyd, J. N. (2008). The time paradox: The new psychology of time that will change your life. Nueva York: Free Press. 